\title{
A NOTE ON THE IRREDUCIBILITY OF LEBESGUE MEASURE WITH APPLICATIONS TO RANDOM WALKS ON THE UNIT CIRCLE
}

\author{
TZUU-SHUH CHIANG
}

\begin{abstract}
Let $\mu$ be a probability measure on $R$. We say that a $\sigma$-finite measure $\lambda$ is irreducible with respect to $\mu$ if there does not exist a Borel set $A$ with $\mu(A)$, $\mu\left(A^{c}\right)>0$ such that $\int_{A} \mu\left(A^{c}-x\right) \lambda(d x)=0$. It is well known that the Lebesgue measure $m(d x)$ is irreducible with respect to any discrete measure whose support is $R$. We prove that every absolutely continuous measure is irreducible with respect to any probability measure whose support is $R$ and give an application of this fact to random walks on the unit circle.
\end{abstract}

A well-known property of Lebesgue measure on the real line $R$ is the following: Let $A$ be a Borel set of positive Lebesgue measure and $\left\{\lambda_{i}\right\}_{i=1}^{\infty}$ a countable dense subset of $R$. Then $m\left(\left(\cup_{i=1}^{\infty}\left(A-\lambda_{i}\right)\right)^{c}\right)=0$. (Here, $m$ denotes the Lebesgue measure). In other words, if we try to cover the real line by translating a set of positive Lebesgue measure through a countable dense set, then we will miss at most a set of Lebesgue measure 0 . We can see this fact from a different point of view. Let $\mu$ be a Borel probability measure concentrated on the countable dense set $\left\{\lambda_{i}\right\}_{i=1}^{\infty}$ and a Borel set with $m(A)>0$. Then

$$
\mu(A-x)>0 \text { a.e. } x-m(d x) \text {. }
$$

(Throughout this paper "a.e. $x-m(d x)$ " will mean almost every $x$ with respect to the measure $m$.) To see this, let $x$ be a point such that $\mu(A-x)=0$. Then $\lambda_{i} \notin A-x$ for every $i=1,2, \ldots$, i.e., $x \notin A-\lambda_{i}$ for every $i=1,2, \ldots$. Therefore, $x \notin$ $\cup_{i=1}^{\infty}\left(A-\lambda_{i}\right)$. Since $m\left(\left(\cup_{i=1}^{\infty}\left(A-\lambda_{i}\right)\right)^{c}\right)=0$, we have $\mu(A-x)>0$ a.e. $x-m(d x)$. We say that a $\sigma$-finite measure $\lambda$ is irreducible with respect to $\mu$ if there does not exist a set $A$ with $\lambda(A), \lambda\left(A^{c}\right)>0$ such that $\int_{A} \mu\left(A^{c}-x\right) \lambda(d x)=0$. Then (1) implies that any absolutely continuous measure is irreducible with respect to $\mu$ if $\mu$ is discrete and has support $R$. It seems obvious that (1) should hold for any probability measure $\mu$ whose support is $R$, not only for those discrete ones. But if one tries to prove this seemingly obvious fact using the same technique as we used when $\mu$ is discrete, the problem which will be encountered is that there is no such a fixed countable dense set $\left\{\lambda_{i}\right\}_{i=1}^{\infty}$ to use as when $\mu$ is discrete. In Theorem 1 we give a proof of this fact making essential use of Fubini's theorem. As an application of this, we show in Theorem 3 that a random walk on the unit circle with normalized

Received by the editors February 18, 1981 and, in revised form, May 8, 1981

1980 Mathematics Subject Classification. Primary 60J15; Secondary 46G99.

Key words and phrases. Irreducibility, random walks, ergodicity.

(C)1982 American Mathematical Society 0002-9939/82/0000-1099/\$01.75 
Lebesgue measure as the initial distribution is ergodic if and only if the transition function does not have a lattice distribution.

THEOREM 1. Let $\mu$ be a Borel probability measure on $R$ with the property that $\mu(E)>0$ for every open set $E \subseteq R$. Then every absolutely continuous measure is irreducible with respect to $\mu$.

Proof. It is easy to see that $\mu(A-x)$ is a measurable function in $x$ for every Borel set $A$. Therefore if we let $B=\{x: \mu(A-x)=0\}$, then $B$ is measurable. Consider,

$$
\begin{aligned}
0 & =\int_{B} \mu(A-x) m(d x)=\iint \chi_{B}(x) \chi_{A-x}(y) \mu(d y) m(d x) \\
& =\iint \chi_{B}(x) \chi_{A-y}(x) m(d x) \mu(d y),
\end{aligned}
$$

so $\int \chi_{B}(x) \chi_{A-y}(x) m(d x)=0$ for a.e. $y-\mu(d y)$. That is, $\int \chi_{B \cap(A-y)}(x) m(d x)=0$ for a.e. $y-\mu(d y)$, i.e.,

$$
m(B \cap(A-y))=0 \text { a.e. } y-\mu(d y) .
$$

Since the support of $\mu=R$, we can choose at least a countable dense set $\left\{y_{i}\right\}_{i=1}^{\infty}$ such that (2) holds. Thus $m\left(B \cap\left(\cup_{i=1}^{\infty}\left(A-y_{i}\right)\right)\right)=0$. But $m\left(\left(\cup_{i=1}^{\infty}\left(A-y_{i}\right)\right)^{c}\right)=0$, so $m(B)=0$. Now, let $\lambda$ be an absolutely continuous measure and $A$ a Borel set with $\lambda(A), \lambda\left(A^{c}\right)>0$. Then $\mu(A-x)>0$ a.e. $x-m(d x)$ in $A^{c}$. So $\mu(A-x)>0$ a.e. $x-\lambda(d x)$ in $A^{c}$. This implies $\int_{A^{c}} \mu(A-x) \lambda(d x)>0$.

REMARK. Theorem 1 can easily be generalized to the unit circle.

Let $X_{0}, X_{1}, \ldots$ be a Markov process on the unit circle $S$ with initial distribution $\frac{1}{2 \pi} m(d x)$ and transition function $p(x, d y)$. We say that $X_{0}, X_{1}, \ldots$ is a random walk if $p(x, d y)=p(d y-x)$. We say that $p(x, d y)$ has a lattice distribution if there exist $x_{1}, \ldots, x_{n}$ such that each $x_{i}$ is a rational multiple of $2 \pi$ and $p\left(0,\left\{x_{i}\right\}_{i=1}^{n}\right)=1$. For an arbitrary random walk, let $\mu=\sum_{n=1}^{\infty} p^{n}(0, d y) / 2^{n}$ where $p^{n}(x, d y)$ is the $n$th transition function. The following lemma is easy and the proof will be omitted.

LEMMA 2. Let $p(x, d y)=p(d y-x)$ be a transition function and $\mu$ be defined as above. Then the support of $\mu$ is $S$ if and only if $p(x, d y)$ does not have a lattice distribution.

A theorem regarding the ergodicity of random walks reads as follows: a random walk on the unit circle with initial distribution $\frac{1}{2 \pi} m(d x)$ is ergodic if and only if there does not exist a set $A$ with $m(A), m\left(A^{c}\right)>0$ such that $\int_{A} p\left(x, A^{c}\right) m(d x)=$ $\int_{A^{c}} p(x, A) m(d x)=0$ (cf. [1, p. 143]). We are now ready to state

THEOREM 3. A random walk on the unit circle with initial distribution $\frac{1}{2 \pi} m(d x)$ and transition function $p(x, d y)$ is ergodic if and only if $p(x, d y)$ does not have a lattice distribution.

Proof. Suppose $p(x, d y)$ does not have a lattice distribution. If the random walk were not ergodic, then there exists a set $A$ with $m(A), m\left(A^{c}\right)>0$ such that 
$\int_{A} p\left(A^{c}-x\right) m(d x)=0$. This implies that $\int_{A} p^{n}\left(A^{c}-x\right) m(d x)=0$ for $n=$ $1,2, \ldots$. Therefore,

$$
\int_{A} \sum_{n=1}^{\infty} \frac{1}{2^{n}} p^{n}\left(A^{c}-x\right) m(d x)=0,
$$

i.e., $\int_{A} \mu\left(A^{c}-x\right) m(d x)=0$. But by Theorem 1 and Lemma 2 ,

$$
\int_{A} \mu\left(A^{c}-x\right) m(d x)>0,
$$

so the random walk is ergodic. The converse is trivial and we omit the proof.

\section{REFERENCES}

1. R. B. Ash and M. F. Gardner, Topics in stochastic processes, Academic Press, New York, 1975.

Department of Mathematical Sciences, University of Wisconsin, Milwaukee, Wisconsin 53201 